\title{
Review
}

Xuemei Li, Xiaoxia Li*

\section{Progress in the treatment of fungal infections of the central nervous system}

DOI: $10.1515 / \mathrm{ii}-2017-0161$

Received December 06, 2017; accepted December 27, 2017; published online April 10, 2018

\begin{abstract}
The incidence of fungal infections of the central nervous system (CNS) has gradually increased in recent years. Intracranial fungal infection can be classified as diffuse and focal infections. The clinical manifestations of these infections include fever and cranial pressure caused by meningitis or meningoencephalitis, as well as focal neurological defects caused by lesions in the intracranial space. Diagnosing fungal infections of the CNS requires comprehensive analysis of the patient's medical history, epidemiology, underlying disease, clinical manifestation, imaging manifestations, and results from various laboratory tests. The identification of fungal bodies or structures in brain tissue or cerebrospinal fluid specimens is the golden standard of diagnosis. The principles for the treatment of fungal infections of the CNS are the effective control of pathogenic risk factors, use of effective antifungal drugs, and the active implementation of surgical intervention for fungal abscesses and granulomas. In the meantime, new diagnoses and treatments should be actively explored to improve the prognosis of patients.
\end{abstract}

Keywords: fungus, infection, central nervous system, treatment, diagnosis

\section{Introduction}

The incidence of fungal infections of the central nervous system (CNS) has gradually increased in recent years [1,2]. Human immunodeficiency virus (HIV) infection, extended hormone and immune inhibitor treatments, diabetes, environmental factors, and age are the high-risk factors for fungal infection [2-4]. Diagnosis and treatment of intracranial fungal infection are difficult and hinder the control of these infections. Patients with intercranial fungal infections have poor prognosis. This paper reviews the progress in the diagnosis and treatment of fungal infections of the CNS.

\section{Fungal pathogens and infection routes of fungal infections of the CNS}

The common fungal pathogens that cause intracranial infections include Candida, Aspergillus, Mucor, Cryptococcus saccharomycetes, Nocardia brasiliensis, and Histoplasma. Intracranial infection is classified as diffuse or focal. Diffuse infection is characterized by meningitis, is mainly caused by Cryptococcus and Candida albicans, and is localized in the macrospore and capsular tissue. Focal infection mainly manifests as granuloma and abscess and is caused by Aspergillus, Candida, zygomycetes, and some species of black fungus. Infections by filamentous fungi, particularly brain abscesses and granulomas caused by Aspergillus, are common and require surgical intervention.

Aspergillus is a common genus of saprophytic fungi and is commonly found in moldy food, grain products, and other moldy organic matter. The most common species of Aspergillus are Aspergillus fumigates,

Department of Rheumatology, Xuanwu Hospital, Capital Medical University, Beijing 10053, China

*Correspondence: Xiaoxia Li, E-mail: lixiaoxia1958@126.com 
Aspergillus terreus, Aspergillus niger, and Aspergillus flavus [5]. Aspergillus flavus commonly infects individuals with normal immune function, whereas Aspergillus fumigates usually infects those with low immunity. The common infection routes are as follows: 1) direct invasion: to invade the intracranial space, Aspergillus destroys the thin bone wall of the skull, thus causing infection; 2) blood infection: fungal sepsis is caused by other factors, such as lung infection, intravenous drug use, and heart surgery. The fungus invades the intracranial space through the blood stream; 3) other routes: infections that are caused by subsequent to craniotomy, open cranial trauma, or lumbar puncture. Reports of these cases, however, are few [6].

\section{Clinical manifestations}

The clinical manifestations of fungal infection can be categorized as diffuse or focal. Diffuse CNS symptoms are caused by meningitis and meningoencephalitis, and focal symptoms of neurological deficits are caused by space-occupying lesions. The most common clinical manifestations of the former are fever and intracranial hypertension.

Mycotic infection seldom causes pure meningitis, mostly manifesting as granuloma or abscess, and usually occurs in 30- to 50-year-old patients. The duration of its symptoms, which ranges from days to months and years, differs in accordance with the progress and timely diagnosis of the disease. The clinical manifestation of intercranial fungal infection is roughly divided into three categories: 1) Involvement of the cranial nerve and basicranial bone wall. The patients present protopathy symptoms such as nasosinusitis, mastoiditis, and orbital periarthritis. 2) Focal neurologic deficit symptoms, which are related to space-occupying lesions. 3) Stroke symptoms, of which the major manifestation is sudden hemiparesis; this symptom, however, is rare [7]. In addition, the patients also experience increased intracranial pressure, epilepsy, and paresthesia. Fever is rare; otherwise, some patients only experience low-grade fever. Only $10 \%-31 \%$ of patients with Aspergillus infection experience fever [2].

\section{Diagnosis and differential diagnosis}

Diagnosing fungal infections of the CNS is comparatively difficult and requires comprehensive analysis of the patient's medical history, epidemic disease, underlying diseases, clinical manifestation, imaging manifestation, and the result of each laboratory test. Although finding fungal bodies or structures in the brain tissue or cerebrospinal fluid (CSF) remains the gold standard for definitive diagnosis, only one-third to half of all patients have positive CSF culture.

Current measures for the clinical diagnosis of fungal infections of the CNS include the following:

1. Conventional and biochemical examination of the CSF: The intracranial pressure of the patients usually increases; cell numbers and protein levels increase; and glucose and chloride levels are normal or lower. However, this examination is not specific. Moreover, the CSF of patients infected with Aspergillus can be normal.

2. Pathogenic test of the CSF: This method includes a smear test under microscopy and a bacterial culture of CSF. Viewing the CSF smear under microscopy can reveal characteristic hyphae and conidia when there is a large amount of fungi present, but this test has a low positive rate. The positive rate of CSF culture is also low.

3. Immunologic test: Using the immune method to directly test for fungal cell wall and cytoplasmic antigens in the CSF is the most direct method for the diagnosis of deep invasive fungal infection [8].

a. Aspergillus galactomannan detection (GM experiment): Galactomannan is a specific heat-stable polysaccharide found in the walls of Aspergillus hyphae. This method can be specific for Aspergillus infection but has a high false-positive rate. 
b. (1,3)- $\beta$-D-glucan detection ( $\mathrm{G}$ test): Except for Cryptococcus and zygomycetes, the cell walls of all fungal species contain (1,3)- $\beta$-D-glucan. Therefore, this method can be used to verify fungal infection. Simultaneously performing the GM and G tests can improve the sensitivity and specificity of Aspergillus detection.

c. Antibody detection: The amount of antibodies in the CSF is greater than that of antigens and is not influenced by empirical antifungal therapy. However, the antibody test cannot distinguish between active or obsolete infection. Some current research and the development of new methods can be used to determine whether fungal infection is active, so the antibody test is expected to become a new diagnostic method.

4. Molecular biology test: This method, which has not been widely applied in the clinic, is used to detect the genomic DNA or RNA fragments of fungal strains. This method remains a highly promising diagnostic method.

5. Imageological examination: Computed tomography (CT) and magnetic resonance (MR) imaging techniques provide the most meaningful image-based examination of fungal infections, particularly mycotic infection, of the CNS [9,10]. In diagnostic imaging, mold abscesses manifest as low T1 signal and high T2 signal. In T2-weighted images of Aspergillus infection, an irregular, circular low signal is present between the abscess wall and the central necrosis area. A low signal indicates the active propagation of Aspergillus [10,11]. During growth, abscessed lesions associated with fungal infection display irregular, discontinuous, thick wall ring reinforcements. Some clinicians refer to the "open loop" as the characteristic presentation of intercranial fungal infection [12]. Some scholars propose initiating treatment with an experimental antifungal drug upon the imaging of characteristic signals [13].

6. Relevant blood tests: Antigen and antibody tests, as well as blood fungal smear and culture, have similar significance as CSF smears. Positive tests also can provide the basis for identifying the route of intracranial fungal infection. Nevertheless, these tests have low positive rates.

\section{Treatment principles}

Treatment principles for fungal infections of the CNS include the effective control of pathogenic risk factors, the application of antifungal drugs, and active surgical intervention in the case of fungal abscess and granuloma.

1. The effective control of pathogenic risk factors: Actively enhance the immunity of the patients; remove the potential sources of infection, such as drainage tubes and deep venous cannula, in a timely manner; focus surgical treatment on infection around the eye pits and paranasal sinus; and sufficiently flush the sinus cavity with antifungal drugs.

2. The application of antifungal drugs: Common antifungal drugs are classified into five categories. Most antifungal drugs affect the cell wall and membrane of the fungus. Antifungal drugs such as polyenes, azoles, propylene amine, and spines ball essence, as well as pyrimidines, which is represented by 5 -fluorouracil, exert antifungal effect by blocking DNA synthesis and inhibiting metabolism.

Voriconazole is the first choice for the treatment of cerebral aspergillosis; high doses of amphotericin B lipid preparations are the second choice. Joint surgery is adopted when necessary [5]. Aspergillus goes against the drug to reach the infection site; therefore, the surgical removal of lesions along with high doses of amphotericin (the daily maximum dose can reach $2 \mathrm{mg} / \mathrm{kg}$ ) is recommended for the treatment of Aspergillus infections. The antifungal drugs voriconazole, posaconazole, or caspofungin, with the surgical removal of lesions, are also recommended for treatment [14].

3. Active surgical intervention: Although surgery is usually performed to diagnose intercranial Aspergillus infection, it can also have therapeutic effects. The common surgical interventions are as follows:

a. Stereotactic techniques: These techniques are usually applied as treatment. Deep lesions at one or more functional sites can be sampled via aspiration by needle biopsy. The samples can then be 
examined under microscopy and cultured for the definite identification of the pathogen. This technique can be used to inject the appropriate antifungal drug into the lesion site.

b. Craniotomy: Craniotomy can be performed when an intracranial lesion suspected as a possible fungal mass is in an accessible location and can be radically excised. However, if the lesion is located in a functional area, radical resection will cause obvious neurological impairment [2].

c. Other surgical intervention measures: Hydrocephaly can be treated with the placement of a ventriculoperitoneal shunt pipe. Fungal aneurysm patients require antifungal treatment, coupled with surgical intervention or interventional embolization at the same time. These patients usually have a bad prognosis.

\section{Prognosis}

Fungal infection of the CNS is characterized by severe neurological symptoms, difficulty in diagnosis and treatment, high mortality rates, and bad prognosis. The prognosis of patients is related to the effectiveness and timeliness of diagnosis and treatment and is closely connected with their immunity. Therefore, early diagnosis, active intervention, and improving immunity can improve the prognosis of patients [15].

\section{Prospect}

Fungal infections of the CNS are rarer than bacterial infections of the CNS. Methods for the diagnosis of intercranial fungal infections have slowly developed slowly and lack standardized criteria. Therefore, more advanced diagnostic methods for this disease require further exploration. The development of new drugs will improve the effectiveness of treatment methods for fungal infections of the CNS. There is no consensus, however, on the surgical treatment, measurement, and scope for fungal treatment. To summarize, fungal infection of the CNS is difficult to diagnose and treat and is associated with poor prognosis. Treatment measures should be further researched and developed to improve the prognosis and to enhance the quality of life of patients.

Acknowledgement: None.

Conflict of interest: The authors state no conflicts of interest.

Authors' Contributions: Xuemei Li and Xiaoxia Li conducted the literature analysis and further wrote, discussed, and revised the manuscript of this review.

\section{References}

[1] Raparia K., Powell S.Z., Cernoch P., Takei H., Cerebral mycosis: 7-year retrospective series in a tertiary center, Neuropathology, 2010, 30, 218-223.

[2] Rajshekhar V., Surgical management of intracranial fungal masses, Neurol. India, 2007, 55, 267-273.

[3] Tahin B., Cooke J., Grasselly M., Manifestations of systemic mycoses and related infections in the central nervous system, Orv. Hetil., 2009, 150, 1367-1374.

[4] Khandelwal N., Gupta V., Singh P., Central nervous system fungal infections in tropics, Neuroimaging Clin. N. Am., 2011, 21, 859-866.

[5] Karthaus M., Guideline based treatment of invasive aspergillosis, Mycoses, 2010, 53, 36-43. 
[6] Genzen J.R., Kenney B., Central nervous system Aspergillus infection after epidural analgesia: diagnosis, therapeutic challenges, and literature review, Diagn. Microbiol. Infect. Dis., 2009, 65, 312-318.

[7] Sharma B.S., Khosla V.K., Kak V.K., Banerjee A.K., Vasishtha R.K., Prasad K.S., et al., Intracranial fungal granuloma, Surg. Neurol., 1997, 47, 489-497.

[8] Fan J., He J., Wu D., et al., Clinical manifestation and laboratory diagnosis of common fungal infection diseases of the central nerve system, J. Brain Nerv. Dis., 2010, 18, 473-476.

[9] Jiménez Caballero P.E., Mollejo Villanueva M., Cerebral aspergilloma in an immunocompetent patient: radiological and histopathological findings, Neurologia, 2009, 24, 275.

[10] Ferrés G.M., Bidart H.T., Zubieta A.M., Importance of images and etiological diagnosis of central nervous system involvement in immunocompromised patient, Rev. Chilena Infectol., 2010, 27, 541-543.

[11] Misra B.K., Guptaa S., Imaging findings in intracranial fungal disease, World Neurosurg., 2010, 74, 581-582.

[12] Chen Y., He Y., Li W., et al., CT and MRI manifestation of the intracranial fungal infection, J. Imaging Intervention, 2010, 7, 66-68.

[13] Siddiqui A., Bashir S.H., Ali Shah A., Sajjad Z., Ahmed N., Jooma R., Diagnostic MR imaging features of craniocerebral Aspergillosis of sino-nasal origin in immunocompetent patients, Acta Neurochir. (Wien), 2006, 148, 155-166.

[14] Redmond A., Dancer M., Woods. Fungal infections of the central nervous system: a review of fungal pathogens and treatment, Neurol. India, 2007, 55, 251-259.

[15] Shamim M.S., Enam S.A., Ali R., Anwar S., Craniocerebral aspergillosis: a review of advances in diagnosis and management, J. Pak. Med. Assoc., 2010, 60, 573-579. 